\title{
On the proton flux towards the plasma grid in a $R F$-driven negative hydrogen ion source for ITER NBI
}

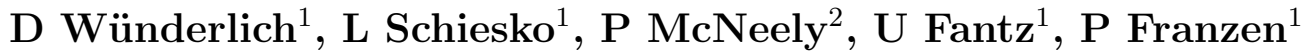 \\ and the NNBI-Team ${ }^{1}$ \\ ${ }^{1}$ Max-Planck-Institut für Plasmaphysik (IPP), EURATOM Association, \\ Boltzmannstr. 2, D-85748 Garching, Germany \\ ${ }^{2}$ Max-Planck-Institut für Plasmaphysik (IPP), EURATOM Association, \\ Wendelsteinstr. 1, D-17491 Greifswald, Germany \\ E-mail: dirk.wuenderlich@ipp.mpg.de
}

\begin{abstract}
The transport of protons in a RF driven negative hydrogen ion source for ITER NBI was investigated by means of a 3d Monte Carlo transport code. As input for the code a consistent axial profile of the electrostatic potential for the complete ion source was constructed. The potential difference between the plasma generation volume and the plasma grid is in the range of 10 to $20 \mathrm{~V}$ and depends on the RF power, the filling pressure and the plasma grid bias voltage. The mean free path for proton collisions with the background gas is in the range of several centimeters which means that the protons are noticeably decelerated and scattered. The velocity distribution function of the protons impinging the plasma grid resembles much more a low-temperature Maxwell distribution function than the high energetic proton beam which would be expected by looking only at the maximum value of the potential in the plasma generation volume. This finding is of high relevance for further analysis of the negative hydrogen ion production on the plasma grid surface as well as the ion transport and beam formation.
\end{abstract}




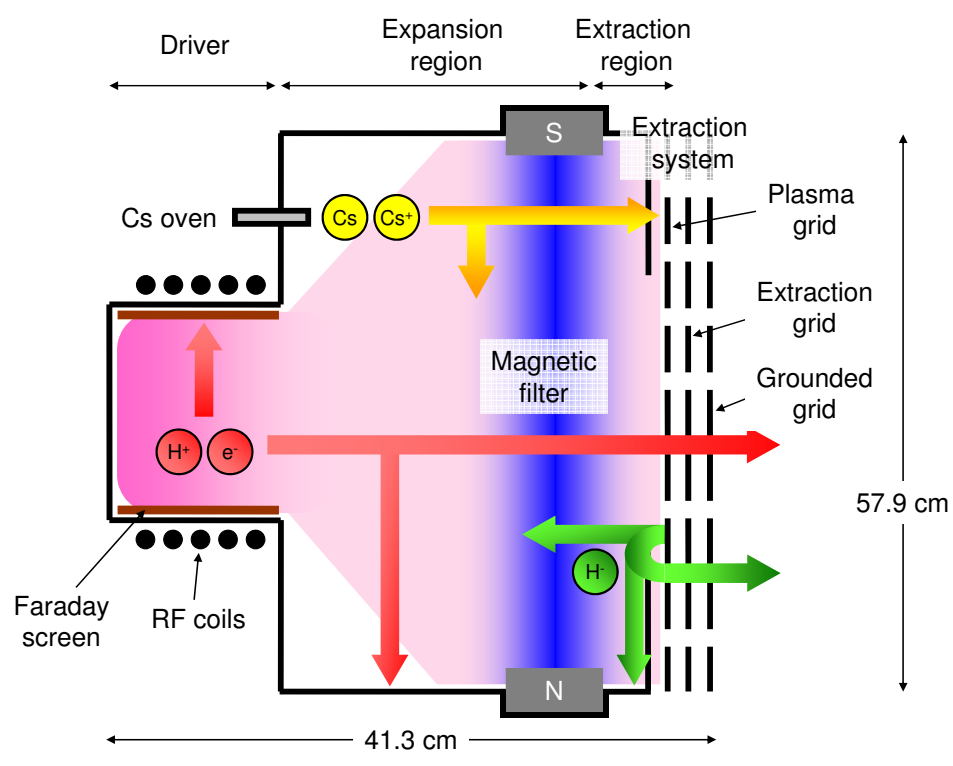

Figure 1. Schematic view of the IPP negative hydrogen ion source prototype.

\section{Introduction}

For heating and current drive in ITER two NBI beamlines are planned with a heating power of 16.5 MW each [1,2]. Each beam line is based on a large source for negative hydrogen ions $\left(A_{\text {source }}=1.9 \times 0.9 \mathrm{~m}^{2}, 1280\right.$ extraction apertures, $\left.A_{\text {extr }}=0.2 \mathrm{~m}^{2}\right)$. The negative ions are accelerated in five stages to a total kinetic energy of $1 \mathrm{MeV}\left(I_{\mathrm{acc}}=40 \mathrm{~A}\right)$ and then neutralized. Since 2007 the RF source for negative hydrogen ions developed at IPP Garching [3, 4] is the ITER baseline design [1]. Currently, at IPP Garching the ELISE test facility $[5,6]\left(\frac{1}{2}\right.$ area of the ITER ion source) is used in order to demonstrate the scalability of the small ( $\frac{1}{8}$ area of the ITER ion source) ion source prototype to larger areas. Finally, a full ITER area ion source [7] and a complete beam line [8] will be constructed at Consorzio RFX in Padua (start of operation is foreseen for 2015 and 2017, respectively).

Figure 1 shows a schematic side view of the IPP prototype ion source. The plasma is generated inductively ( $f_{\mathrm{RF}}=1 \mathrm{MHz}$, typical $\mathrm{RF}$ power: 70 to $100 \mathrm{~kW}$ ) in the driver and then expands into the expansion region. In order to reduce the destruction rate of negative ions and the amount of co-extracted electrons the electrons are cooled down (to $T_{\mathrm{e}} \approx 1 \mathrm{eV}$ at electron densities around $10^{17} \mathrm{~m}^{3}$ ) close to the extraction system by means of a magnetic filter field. The extraction and acceleration system consists of three grids: the plasma grid, the extraction grid and the grounded grid. In order to reduce the ratio of co-extracted electrons to extracted negative ions the plasma grid is positively biased with respect to the source body. The co-extracted electrons are removed from the extracted negative ion beam by means of electron deflection magnets embedded into the extraction grid.

The production of negative hydrogen or deuterium ions occurs mainly at the surface 
of the plasma grid, which is covered with a thin cesium layer in order to reduce the surface work function $[3,9,10]$. This production process is based on the transfer of electrons from the surface to approaching hydrogen atoms [11] or positive hydrogen ions [12]. The latter are neutralized in a first step by an Auger process close to the surface; molecular ions are dissociated first. The surface generated negative ions initially move towards the plasma volume. Their trajectory is redirected towards the plasma grid extraction apertures by interaction with the magnetic filter, the electron deflection field and by charge exchange collisions [13].

The neutral gas expansion into the ion source can be assumed to be homogeneous before and after the plasma pulses. However, during the pulses the gas distribution is influenced by neutral depletion [14]. Although no spatially resolved measurements exist, some effect of neutral depletion on the homogeneity of the atomic hydrogen density close to the plasma grid can be expected. On the other hand, the plasma and thus also the positive hydrogen ion flux in front of the extraction system is non-uniform [15]. Reason is a vertical plasma drift, caused by the magnetic filter field. The degree of this non-uniformity can be influenced over a wide range by the bias voltage [16].

Since the described non-uniformity of the gas and the plasma are caused by different physical effects, the spatial distribution of the atomic hydrogen density over the plasma grid surface is not necessarily identical to the one of the plasma density. As a consequence, the overall homogeneity of the negative ion production and in turn also the homogeneity of the extracted negative ion beam is strongly correlated to the spatial distribution of the relative relevance of the atomic and positive ion production channel for negative hydrogen ions. This is of particular importance since for ITER NBI uniformity of the beam better than $10 \%$ is required.

In [17] it was shown that for low temperature positive ions and atoms $\left(\mathrm{T}\left(\mathrm{H}_{x}^{+}\right)=\right.$ $\mathrm{T}(\mathrm{H})=0.8 \mathrm{eV}$ ) close to the plasma grid the negative ion flux created from conversion of atoms is by a factor of 4 larger than the negative ion flux created from conversion of positive ions. The assumption of low temperature Maxwell velocity distribution functions for the hydrogen atoms and protons is based on the following experimental and theoretical results: Firstly, measuring the Doppler broadening of the $\mathrm{H}_{\alpha}$ line gives an upper limit for the temperature of atomic hydrogen of $0.8 \mathrm{eV}$ [18]. Secondly, Langmuir probe measurements indicate that the proton temperature close to the plasma grid is comparable to the atomic temperature while $T\left(\mathrm{H}_{2}^{+}\right)$and $T\left(\mathrm{H}_{3}^{+}\right)$are significantly smaller [19]. Thirdly, measurements with a Mach probe show that the flow velocity of positive ions in the extraction region is relatively small: $\approx 10^{2} \frac{\mathrm{m}}{\mathrm{s}}$ with magnetic filter field, $\approx 10^{4} \frac{\mathrm{m}}{\mathrm{s}}$ without magnetic filter field [20]. These Mach probe results have been confirmed by means of a simple flow code [21]. Additionally, it was demonstrated by means of beam emission spectroscopy that the negative hydrogen ion beam is much more homogeneous compared to the plasma non-uniformity [22], indicating a high relevance of the negative ion production by conversion of atoms.

In contrast to the above mentioned experimental and theoretical results, a recent publication [23] claims that the mean free path for collisions of positive ions generated 
in the driver is larger than the dimension of the IPP prototype ion source. Thus, it is stated that these positive ions are accelerated without collisions towards the plasma grid, resulting in the presence of an ion beam with particle energies of several ten $\mathrm{eV}$. The consequences on the physics of the production and extraction of negative hydrogen ions (e.g. ion survival probability and beam divergence) would be immense: Conversion of positive ions would be the by far dominant negative hydrogen ion production channel. The beam homogeneity would be determined solely by the plasma homogeneity and would be correlated to the applied bias voltage - which is not the case. Additionally, since the kinetic energy of the negative ions is a decisive parameter for their survival probability during the transport to the extraction apertures [13] as well as for the divergence of the extracted ion beam $[24,25,26]$, the current picture of the negative ion transport and beam formation would have to be reconsidered.

In order to solve the raised contradiction, investigations on the transport of positive ions from the driver to the plasma grid surface have been undertaken. Aim was to determine the energy of the positive hydrogen ions striking the plasma grid surface. The first step was to gather the available experimental results on the electrostatic potentials in the prototype ion source in order to obtain a consistent axial potential profile from the driver to the plasma grid. Then, this potential profile is used as input for a transport code in order to estimate the collision probabilities of positive ions generated in the driver on their way towards the plasma grid. In order to reduce the complexity, these investigations are restricted to the transport of protons. The final step is to simulate the transport inside the ion source volume for an ensemble of protons and calculate the energy distribution of the protons impinging on the plasma grid surface, as a function of different source parameters.

\section{The code ProtonFlow3d}

In order to investigate the transport of protons generated in the driver of the IPP prototype negative ion source to the plasma grid the three dimensional transport code ProtonFlow3d was developed. The code is a test particle Monte Carlo code. In it, single protons are created at a defined position (or in a defined volume) in the driver. The code then follows the motion of the particle in the background plasma until it hits one of the inner surfaces of the ion source. The plasma grid surface is treated as a regular inner surface, i.e. it is not distinguished between the extraction apertures and the area in-between the apertures. There is no need to implement the full $3 \mathrm{~d}$ structure of the plasma grid with its extraction apertures since the meniscus shaped extraction surfaces do not penetrate deeply into the plasma volume [27] and thus do not influence the proton trajectories in the bulk plasma.

The motion of the test particles is divided into small time steps. For each time step the electrostatic field strength $\vec{E}$ (calculated from the profile of the potential in the source plasma) is substituted into the Lorentz equation in order to calculate the particle 
Table 1. Reaction processes implemented to ProtonFlow3d

\begin{tabular}{cccc}
\hline Reaction & Process & Ref. & Comment \\
\hline MT with $\mathrm{H}$ & $\mathrm{H}^{+}+\mathrm{H} \rightarrow \mathrm{H}^{+}+\mathrm{H}$ & {$[29]$} & \\
MT with $\mathrm{H}_{2}$ & $\mathrm{H}^{+}+\mathrm{H}_{2} \rightarrow \mathrm{H}^{+}+\mathrm{H}_{2}$ & {$[30]$} & \\
CX with $\mathrm{H}$ & $\mathrm{H}^{+}+\mathrm{H} \rightarrow \mathrm{H}+\mathrm{H}^{+}$ & {$[31]$} \\
CX with $\mathrm{H}_{2}$ & $\mathrm{H}^{+}+\mathrm{H}_{2} \rightarrow \mathrm{H}+\mathrm{H}_{2}^{+}$ & {$[31]$} & $T_{\text {vib }}=5000 K$ \\
Vib. excitation & $\mathrm{H}^{+}+\mathrm{H}_{2}(v=0) \rightarrow \mathrm{H}^{+}+\mathrm{H}_{2}(v=1 \ldots 3)$ & {$[30]$} & \\
Rot. excitation & $\mathrm{H}^{+}+\mathrm{H}_{2}(J=0) \rightarrow \mathrm{H}^{+}+\mathrm{H}_{2}(J=2)$ & {$[30]$} & \\
\hline
\end{tabular}

velocity $\vec{v}^{\prime}$ after the time step:

$$
\vec{v}^{\prime}=\vec{v}+\frac{\vec{E} \cdot q}{m} \Delta t
$$

where $q$ and $m$ are the charge and mass of the test particle, respectively, and $\Delta t$ the time step used for the calculation. The electrostatic field has to be defined externally since test particle codes cannot calculate electrostatic fields in a self-consistent manner.

Additionally, a Monte Carlo module is invoked for each time step in order to decide whether the particle participates in an elastic or inelastic collision. The collision frequencies of all collision processes are summed up to a total collision frequency which in turn is used in connection with the path length estimator algorithm [28] to determine the probability $P_{\text {col }}$ for a collision during a time step:

$$
P_{\mathrm{col}}=1-\exp \left(-\Delta t \cdot \sum_{i} n_{i} \cdot \sigma_{i} \cdot v_{i}\right)
$$

where $n_{i}$ is the density of the collision partner involved in the $i$ th collision process, $\sigma_{i}$ is the corresponding cross section and $v_{i}$ the relative velocity of the two colliding particles. ProtonFlow3d assumes that the collision target is at rest, i.e. the relative velocity $v_{i}$ of the two colliding particles is equal to the absolute value of the proton velocity. Obtaining more realistic relative particle velocities is not straightforward: a flow of gas and plasma particles from the driver towards the plasma grid surface is present in the IPP negative ion sources. Thus, it is not possible to use for the collision partner a random velocity corresponding to a Maxwellian distribution function. A better solution for the neutrals might be a drifting Maxwellian. However, the collision partners of the proton collisions implemented to the code are solely neutral particles (hydrogen atoms and molecules) which are not accelerated in the electrostatic field. Therefore, the effect on the code results caused by neglecting the velocity of the collision partner is assumed to be small.

By comparing $P_{\text {col }}$ with a random number it is determined whether or not during a time step a collision process occurs. If a collision takes place, a second random number is generated. The specific collision that takes place is determined by comparing this random number with the relative contributions of the different single collision processes to the total collision frequency (i.e. the sum of all collision processes) [28]. 


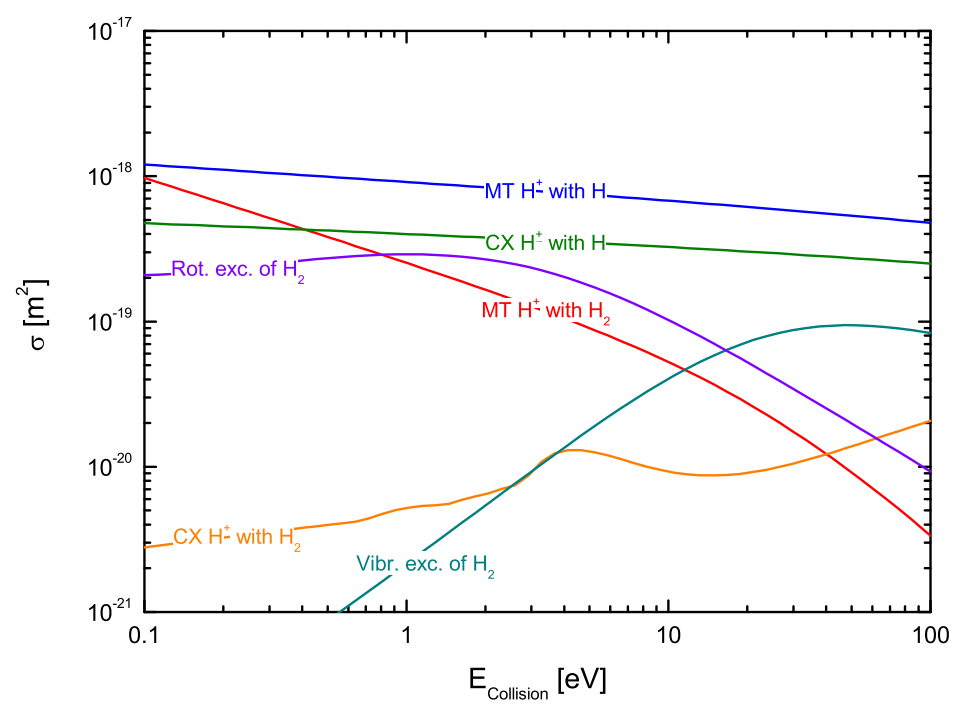

Figure 2. Cross sections for the collision processes implemented to ProtonFlow3d.

Included in ProtonFlow3d are the most relevant reaction processes for protons, i.e. the reactions with the highest collision frequency. These reactions are listed in Table 1. They are charge exchange (CX) collisions, elastic momentum transfer (MT) collisions and two inelastic excitation processes: vibrational and rotational excitation of ground state hydrogen molecules. Figure 2 shows the corresponding cross sections. For the MT reaction of protons with hydrogen atoms the center of mass (CM) energy has to be used for determining the cross section from this figure. For all other reactions the laboratory energy of the proton has to be applied, determined in a reference system in which the collision target is at rest.

The probability for a CX collision of protons with hydrogen molecules depends on the vibrational excitation of the molecule. A strong increase of the cross section with the vibrational quantum number can be seen in the data from [31]. In order to obtain an effective cross section, the cross sections for the different vibrational quantum numbers can be averaged, weighted by the relative population of the vibrational levels. It has been shown by optical emission spectroscopy that it is a good approximation to describe the vibrational population of the hydrogen molecule in the IPP prototype ion source by means of a Maxwellian distribution, characterized by a vibrational temperature. In hydrogen plasmas a value of $4000 \mathrm{~K}$ was measured in the driver (averaged over a line of sight traversing the driver volume axially), almost independent on RF power and bias voltage [32]. In the expansion region (averaged over a line of sight parallel to the plasma grid in $2 \mathrm{~cm}$ distance to the grid surface) a range of vibrational temperatures between $5000 \mathrm{~K}$ and $10000 \mathrm{~K}$ has been measured, depending on the source parameters [32, 33]. For the sake of simplicity, a vibrational temperature of $5000 \mathrm{~K}$ has been used over the complete source volume for implementing CX of protons with hydrogen molecules to ProtonFlow3d.

For rotational and vibrational excitation of hydrogen molecules only the first 
possible excitation steps have been implemented $(v=0 \rightarrow 1 \ldots 3, J=0 \rightarrow 2)$ since only for these excitations steps are cross sections available. The effect of this restriction on the code results is assumed to be negligible since the threshold energy of the implemented reactions is small and thus the cross sections are much larger when compared to the excitation of higher vibrational or rotational levels. Rotational excitation from $J=0$ to $J=1$, which may be presumed as the first possible rotational excitation process, is not allowed since it would require the conversion of ortho-hydrogen to para-hydrogen [34].

During CX collisions, one electron is transferred from the neutral particle to the proton. The relative change in the trajectories of both particles involved in the collision can be described by the repulsive Coulomb interaction between the two nuclei [35]. Since this interaction is weak, usually in Monte Carlo models it is assumed that the trajectories are not changed at all: the velocity of the newly created proton ion is equal to the velocity of the incident atom and vice versa. This scheme is also used in ProtonFlow3d, which means that positive ions generated by CX processes have zero initial kinetic energy. The trajectory of the fast hydrogen atoms generated by the CX process is not followed by the current version of ProtonFlow3d. The presence of these atoms may influence the atomic energy distribution close to the plasma grid, which in turn could result in a slightly modified yield for negative hydrogen ion production by conversion of atoms.

For treating MT collisions the vector of the relative velocity of the two collision partners is determined. Then the procedure described in [36] is applied: the relative velocity is rotated by the two collision angles $\Theta$ and $\Phi$ and then distributed amongst the two collision particles according to the ratio of the particle masses to the reduced mass of the two-particle system. The angle $\Phi$ is chosen randomly between 0 and $2 \pi$. For determining $\Theta$ the following formula (deduced in [37] for elastic ion-neutral collisions) is applied:

$$
\Theta=\arccos (1-2 \cdot \text { Rnd })
$$

where Rnd is a random number between 0 and 1 .

Rotational and vibrational excitation is treated like the MT collisions, with the exception that before redistributing the vector of the rotated relative velocity to the two particles the excitation energy is subtracted. The same formula for the collision angle is applied as for the elastic collisions. This is a simplified approach, ignoring that the collision angle of inelastic collisions is determined by the differential cross section. Data for the differential cross sections for vibrational [38] and rotational [39] excitation of $\mathrm{H}_{2}$ by proton collision are available, but only for a too small number of different collision energies.

For the sake of simplicity Coulomb collisions are neglected by ProtonFlow3d as well as the influence of the magnetic filter field on the proton trajectories. This means that the energies calculated for the protons close to the plasma grid represent an upper limit since Coulomb collisions and the filter will result in a more pronounced deceleration and thermalization of the protons. 
The motion of the test particle is followed until it hits one of the inner surfaces of the ion source. If via a CX process a molecular hydrogen ion is created, the calculation is stopped. In the future ProtonFlow3d will implement following the trajectory of these molecular ions and even the trajectories of the neutral particles involved in the various collision processes.

The geometry routines in the current version of ProtonFlow3d are rather simple: the driver consists of a cylindrical shape of length $17.6 \mathrm{~cm}$ and radius $11.8 \mathrm{~cm}$, attached to a rectangular box with an extent of $23.7 \mathrm{~cm}$ in axial direction of the driver and side lengths of $57.9 \mathrm{~cm}$ and $30.9 \mathrm{~cm}$. These values represent a simplified version of the IPP prototype ion source. After the new position of the test particle for the current time step has been calculated, the code checks whether this position lies inside the source volume or not.

\section{Calculation of proton trajectories}

\subsection{Input Parameters}

For the calculation of collision frequencies of the different collision processes both the velocities of the collision partners as well as the density of the background particle species has to be known. Since the velocity of the neutrals is assumed to be zero, only the velocity of the test particle is necessary, which is the logical outcome of the current simulation process.

The test protons are started in the center of the driver (at $33.2 \mathrm{~cm}$ from the plasma grid) with an initial velocity equal the thermal velocity of $T\left(\mathrm{H}^{+}\right)=0.8 \mathrm{eV}$, headed directly towards the plasma grid. Starting test particles also in the expansion region is not necessary since the electron temperature and electron density in this plasma volume $\left(T_{\mathrm{e}} \approx 1 \mathrm{eV}, n_{\mathrm{e}} \approx 10^{17} \mathrm{~m}^{3}\right)$ are significantly lower than in the driver $\left(T_{\mathrm{e}} \approx 10 \mathrm{eV}\right.$, $\left.n_{\mathrm{e}} \approx 10^{18} \mathrm{~m}^{3}\right)$. Thus, the proton production rate in the expansion region is negligibly small compared to the driver. The choice of $0.8 \mathrm{eV}$ as initial temperature of the protons is justified by the fact that protons are produced in the driver by either dissociative ionization of molecules or charge exchange of $\mathrm{H}$ with $\mathrm{H}_{2}^{+}$or direct ionization of atoms. All these three channels produce protons with an initial energy of around or below $1 \mathrm{eV}$ : from the Franck Condon densities of [40] it can be deduced that the probability for dissociative ionization via the ionic ground state is at its maximum for the production of low energy protons and rapidly decreases for higher proton energy. As already stated in the introduction, $0.8 \mathrm{eV}$ has been determined as upper limit for the atomic temperature by measuring the Doppler broadening of the $\mathrm{H}_{\alpha}$ line in the driver [18].

Similar to [23] the densities of the neutral hydrogen molecules and the ratio of atomic to molecular hydrogen density $\left(n(\mathrm{H}) / n\left(\mathrm{H}_{2}\right)=0.1\right)$ implemented in the code have been taken from [14]. In earlier publications higher atomic densities have been reported, but for a different source configuration and for different source parameters [33]. Thus, the atomic density used for the present calculations constitutes some kind of 
lower threshold and the effect of atomic collisions on the proton collisions may be higher than predicted by ProtonFlow3d. This again means that the proton energies close to the plasma grid calculated by the code represent a upper limit

The last, but most important, input parameter is the electrostatic field inside the negative ion source. In the experiment a complex three-dimensional field structure is present which in principle could be implemented to ProtonFlow3d. Unfortunately, experimental data on the $3 \mathrm{~d}$ field structure is scarce. At present, no code exists that can calculate this structure (for the plasma parameters and particle densities present in the IPP ion sources). Measurements of the potential have been performed mainly in the axial direction (perpendicular to the plasma grid surface) and thus only the axial dependence of the electric field is taken into account by ProtonFlow3d. A full 3d potential map would include the plasma sheath at the ion source side walls as well as potential gradients perpendicular to the axial direction, resulting in an additional deflection of the proton trajectories towards the side walls. The potentials measured by [15] indicate that for the magnetic filter field positioned as close as possible to the plasma grid the perpendicular potential gradients are small compared to the axial potential variation. However, the calculated number of protons reaching the plasma grid surface will be smaller for a full $3 \mathrm{~d}$ potential map, in favor of the protons hitting one of the ion source side walls.

Several Langmuir probe measurements have been performed in the driver and the expansion region of the IPP negative ion source prototype during the last years. Potential profiles have been determined separately for both regions using different probes and for different source and plasma parameters. Up to now, it was not attempted to measure a potential profile for both regions in one go. Hence, the plasma potential profiles used as input for ProtonFlow3d are generated by scaling and merging the results for the driver from [41] and for the expansion region from [15].

The profiles reported in [41] for the driver have been measured for two different sets of source parameters $\left(P_{\mathrm{RF}}=34 \mathrm{~kW}, p_{\text {fill }}=0.75 \mathrm{~Pa}\right.$ and $P_{\mathrm{RF}}=55 \mathrm{~kW}, p_{\text {fill }}=0.27 \mathrm{~Pa}$, both in hydrogen in a non-cesiated source and with the magnetic filter field magnets embedded into the diagnostic flange). Additionally, the influence of the RF power and the filling pressure on the plasma potential was investigated for the driver and the expansion region.

The profiles from [15] for the expansion region were collected at three different positions of the new magnetic filter field frame [42] but only for one set of source parameters $\left(P_{\mathrm{RF}}=40 \mathrm{~kW}, p_{\text {fill }}=0.6 \mathrm{~Pa}\right)$. The measurements have been performed by means of two L-shaped probes introduced into the upper and lower half of the back plate of the expansion region. In order to obtain the potential profile for the center of the source, the results of both probes have been averaged. To ensure comparability with the results from [41], potentials measured in a non-cesiated source have been used.

An additional and very useful piece of information comes from the measured bias voltage (i.e. the voltage of the plasma grid with respect to the other inner surfaces of the ion source). The bias voltage is applied with respect to the experiment ground potential and thus can be compared directly to the potentials measured by the Langmuir probes. 

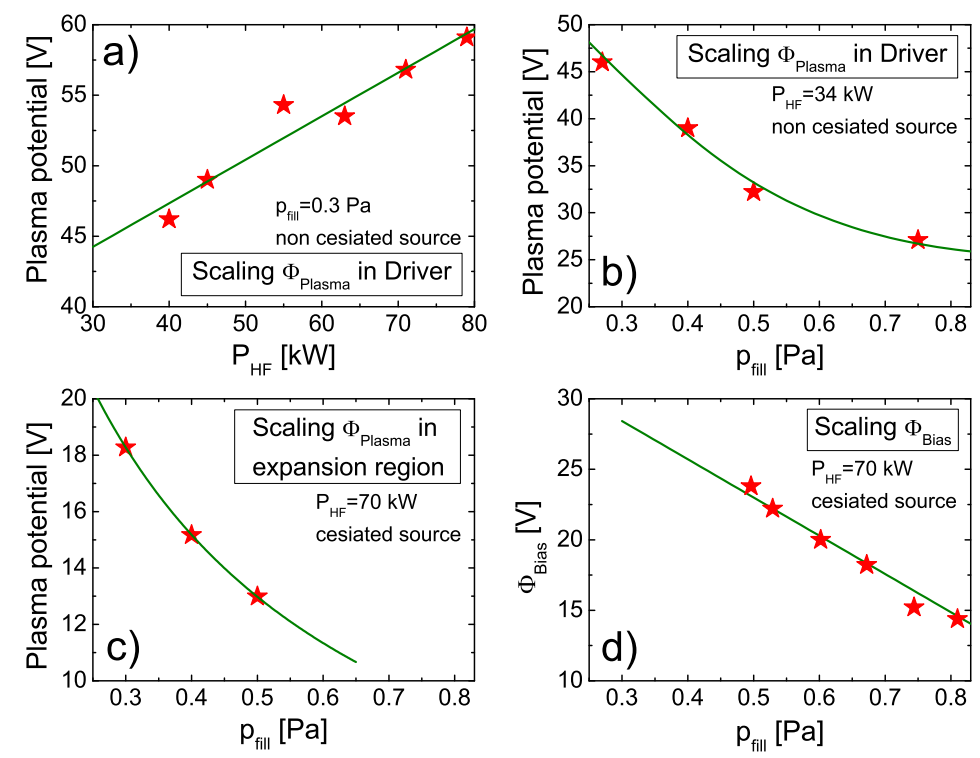

Figure 3. Relations used for scaling measured potentials in order to construct a consistent potential axial profile over the complete source volume. a)-c): relative scaling of the plasma potential, taken from [14], d): relative scaling of the bias potential (self-adjusted in current controlled bias operation).

During most experimental campaigns the bias circuit is current controlled, i.e. the bias voltage is automatically adjusted in order to constantly draw a preset bias current. The value of the bias current is optimized in order to achieve an optimum between suppression of the co-extracted electrons and a high extracted negative hydrogen ion current [43].

Two different scenarios of source operation have been investigated using ProtonFlow3d, both for the magnetic filter field frame positioned as close as possible to the extraction region: the first one $\left(P_{\mathrm{RF}}=40 \mathrm{~kW}, p_{\text {fill }}=0.6 \mathrm{~Pa}\right)$ is one scenario often used for physical operation of the IPP prototype ion source (this pressure is higher compared to the $0.3 \mathrm{~Pa}$ required by ITER, but only for such pressures is it possible to reliably ignite the plasma when the magnetic filter field frame is positioned close to the driver exit). The molecular hydrogen density for these parameters from [41] is $6 \cdot 10^{19} \mathrm{~m}^{-3}$. The bias potential and the potential profile in the expansion region are directly available from measurements. The axial potential profile for the driver under the conditions $P_{\mathrm{RF}}=34 \mathrm{~kW}, p_{\text {fill }}=0.75 \mathrm{~Pa}$ was scaled using the relations reported in [41] and shown in Figure 3 a) and b). The resulting potential profile for low power operation, shown in Figure 4 shows a smooth behavior. The total potential drop seen by a proton generated in the driver $(\approx 7.5 \mathrm{~V})$ is much lower than expected by just looking at the (unscaled) potential values taken from literature as was done in [23].

This figure does not change dramatically for the second set of parameters $\left(P_{\mathrm{RF}}=\right.$ $70 \mathrm{~kW}, p_{\text {fill }}=0.45 \mathrm{~Pa}$ ) which represents operation at higher RF power and lower filling pressure than the first parameter set. The molecular hydrogen density determined in 


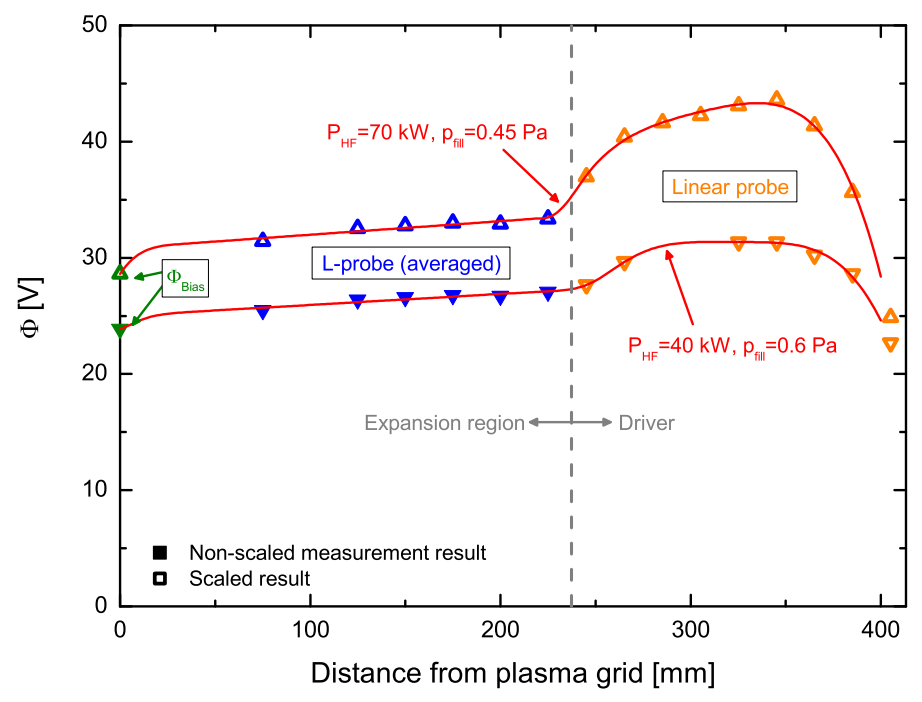

Figure 4. Axial potential profiles for different operation scenarios in the IPP prototype ion source.

[41] for this configuration is $4.5 \cdot 10^{19} \mathrm{~m}^{-3}$. The measured potentials for driver and expansion region as well as the bias voltage had to be scaled (the relations are shown in Figure 3) in order to generate a coherent profile for these source parameters. The profile again looks very smooth which is, of course, no proof but an indication that the performed scaling procedure is applicable. The maximum potential difference between driver and plasma grid is $\approx 14.7 \mathrm{~V}$. This is still much lower than the $45 \mathrm{~V}$ used in the investigations of [23]. The main reason for this huge difference is that in [23] a value of $15 \mathrm{~V}$ is used for the bias potential independent of other source parameters, while in the actual operation of the source the bias voltage strongly depends on the source parameters.

Although the two chosen parameter sets are slightly different compared to the parameters used in [23] $\left(P_{\mathrm{RF}}=55 \mathrm{~kW}, p_{\text {fill }}=0.3 \mathrm{~Pa}\right)$, the potential profiles and the potential differences clearly show that the approach used in [23] (directly comparing potentials measured at different positions inside the ion source for different source configurations and source parameters and neglecting the bias voltage) is not appropriate.

\subsection{Results}

As described earlier, six types of collisions are included in ProtonFlow3d (see table 1). In order to investigate the relative importance of these processes during proton transport, the mean free path $(\mathrm{mfp})$ of the collision processes separately and the total mfp have been calculated for the both cases described above (low and high power operation).

The trajectory of a single test proton created in the driver was followed until it reaches the plasma grid. Any influence of collisions on the proton trajectories has been disabled because it would add a random factor that is undesirable for comparing the mfp with the values used in [23]. The calculated mfp for the separate reactions and the 


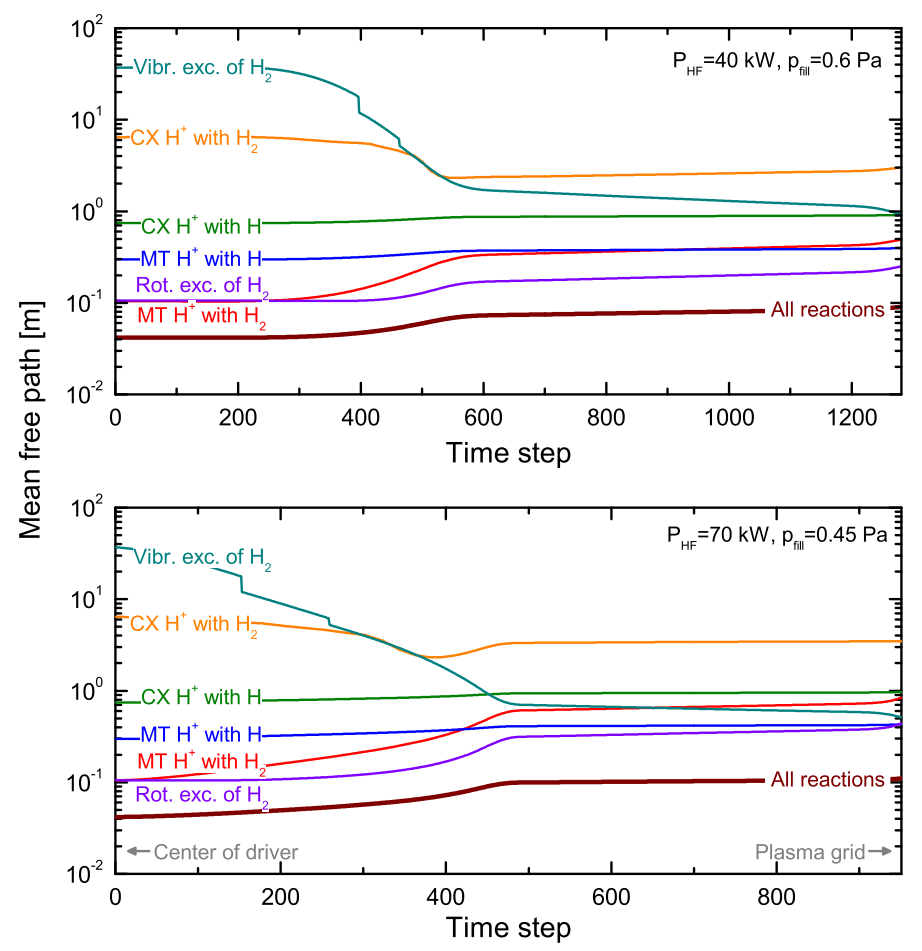

Figure 5. Theoretical mean free path for a test proton for low power operation and the high power scenario. Shown are the mfp for the separate reactions as well as the resulting total mfp.

resulting total mfp are shown in Figure 5 as a function of the time of flight of the proton from its point of birth in the center of the driver to the plasma grid surface. For low power operation the proton reaches the plasma grid after $1.3 \cdot 10^{-5} \mathrm{~s}$ while in the high performance scenario due to the stronger acceleration in the electrostatic field this time is reduced to $9.2 \cdot 10^{-6} \mathrm{~s}$. Two steps can be seen in the mean free path for vibrational excitation. They are caused by the acceleration of the proton to an energy higher than the threshold for exciting the vibrational levels $v=2$ and $v=3$ of $\mathrm{H}_{2}$.

In [23] only two reactions were considered (MT with molecular hydrogen and CX with hydrogen atoms) and the mfp was determined for a fully accelerated particle. The mfp of CX with hydrogen atoms determined by ProtonFlow3d is more or less comparable to the result presented in [23]: $0.71 \mathrm{~m}$ compared to $1.00 \mathrm{~m}$ (for this comparison the values calculated by ProtonFlow3d for the highest particle energy in the high power scenario, i.e. $14.7 \mathrm{eV}$ plus the thermal energy of the proton have been used). In contrast, the mfp for MT with molecules is $0.61 \mathrm{~m}$ which is by a factor of six smaller than the $3.65 \mathrm{~m}$ determined by [23]. Reason for this difference in the calculated mfp is the dependence of the reaction cross section on the proton energy.

For calculating the total mfp of a plasma particle, all reactions have to be taken into account:

$$
\operatorname{mfp}=\frac{v}{\sum_{i} \nu_{i}}
$$


where $v$ is the velocity of the particle, $\nu_{i}$ the frequency of the $i$ th collision process and it is summed over all collisions possible. It is not appropriate to take the mfp of one or two single reaction types and argue that these values are larger than the dimensions of a plasma experiment.

As can be seen in Figure 5, the total mfp for low power operation is $4 \mathrm{~cm}$ in the driver and increases to $8 \mathrm{~cm}$ close to the plasma grid surface. In the high power scenario the mfp for driver and expansion region are $4 \mathrm{~cm}$ and $10 \mathrm{~cm}$, respectively. These values depend on the proton energy but are considerably smaller than the source dimensions. Thus, a much higher collision probability is expected for the protons than predicted in [23].

In order to investigate the effect of this higher collision probability on the energy of the proton impinging the plasma grid, the next step of the investigation is based on the simulation of the trajectories of a large number of test protons, taking into account now interactions with background particles. Shown in Figure 6 are the trajectories of two of these test protons: the first proton (green trajectory) hits the side wall of the ion source while the second one (red trajectory) reaches the plasma grid with a perpendicular kinetic energy $E_{\mathrm{x}}$ of $2.34 \mathrm{eV}$.

Calculations have been performed for an ensemble of $10^{5}$ protons. Shown in Figure 7 is the statistics of the points at which the protons hit the inner surfaces of the ion source. For low power operation $48.1 \%$ of the protons hit the plasma grid, $23.8 \%$ the driver back plate and $11.7 \%$ the side walls of the expansion region. As can be seen in Figure 4, the potential profile inside the volume of the driver for the high power scenario shows a pronounced gradient while the potential for low power operation is flatter over that area. As a result, in the high power scenario the force that drives the protons out of the driver is stronger, resulting in a dramatically smaller probability for the protons to hit the driver back plate or the driver side walls $(0.1 \%)$. Consequently, the number of protons reaching the plasma grid is significantly higher for the high power scenario $(63.0 \%)$ than for low power operation.

The main question is: what is the energy distribution of the protons hitting the plasma grid in either scenario? The top part of Figure 8 shows the calculated distribution of the kinetic energy (more precisely: the energy component $E_{\mathrm{x}}$ perpendicular to the grid surface). Also shown for comparison is a 1d Maxwell energy distribution for $T\left(\mathrm{H}^{+}\right)=0.8 \mathrm{eV}$.

The calculated proton energy distributions show a broad peak in both scenarios. Compared to the 1d Maxwell distribution for $T\left(\mathrm{H}^{+}\right)=0.8 \mathrm{eV}$ the distribution function is shifted to slightly higher energies. The maximum of the broad peak is at $1.4 \mathrm{eV}$ for low power operation and is at $2.6 \mathrm{eV}$ for the high power scenario. This peak is the result of the beginning of the thermalization process.

For higher energies a weak continuum is observed, culminating in sharp peaks which represent the protons that have experienced no collision. The observed energy of the peaks is equal to the potential drop between driver and plasma grid plus the $0.8 \mathrm{eV}$ starting energy of the proton. The area of the peaks give the percentage of protons 


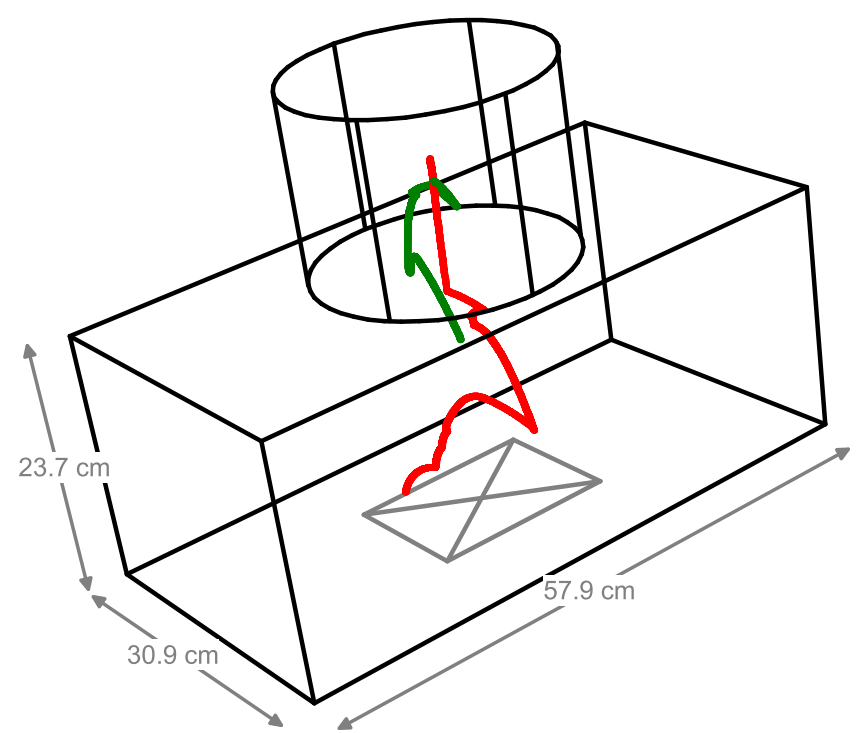

Figure 6. Two example trajectories. The green particle hits the side wall of the negative ion source, the red one reaches the plasma grid surface with a kinetic energy of $E_{\mathrm{x}}=2.34 \mathrm{eV}$.
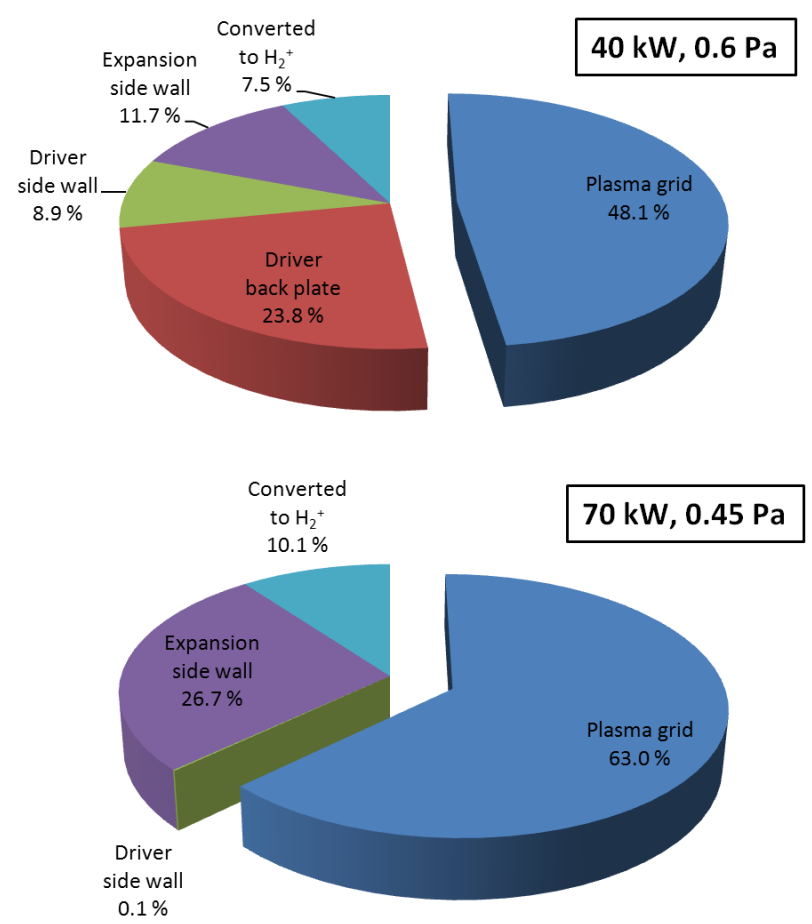

Figure 7. Statistics of the proton impact points for the two considered scenarios. 


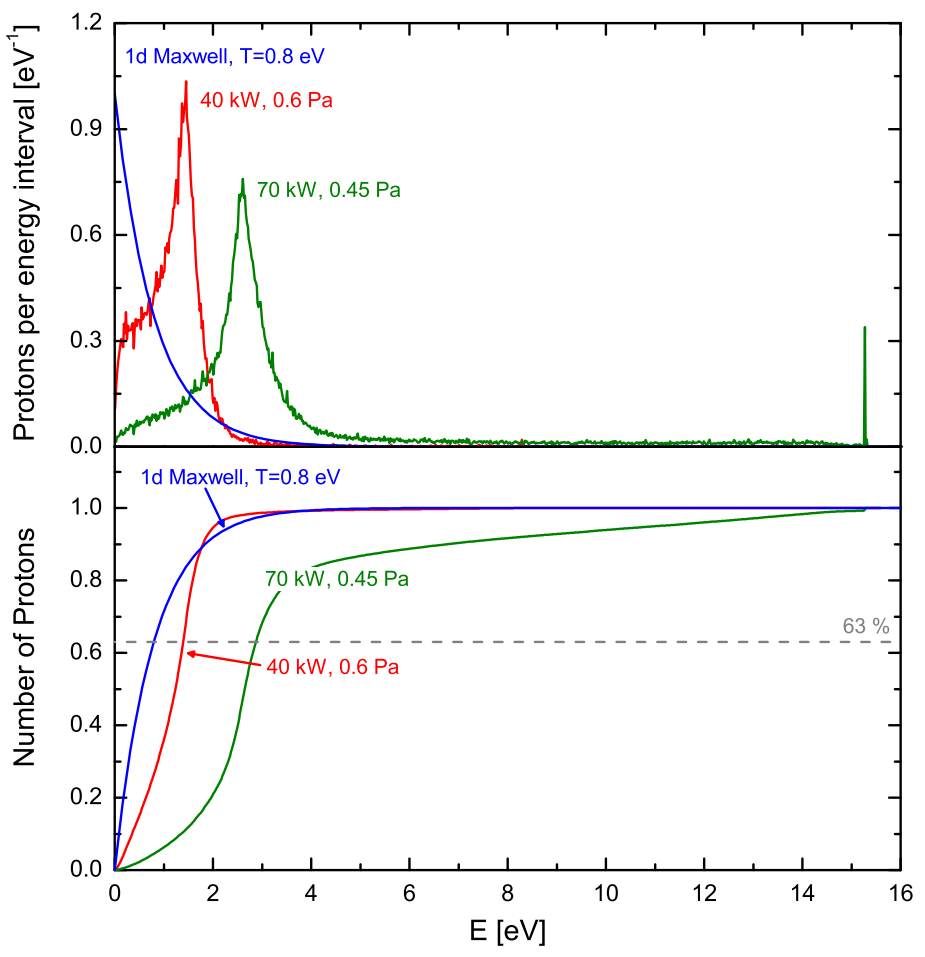

Figure 8. Energy distribution of the protons impinging the plasma grid surface (top). Integrated energy distribution (bottom).

which do not collide during their transport to the plasma grid: a negligible fraction in low power operation (the peak at $8.3 \mathrm{eV}$ is almost undetectable) and approximately $0.4 \%$ in the high power scenario (peak at $15.5 \mathrm{eV}$ ).

Shown in the bottom part of Figure 8 is the integral of the energy distribution calculated for the two operation scenarios. These curves give the percentage of protons that hit the plasma grid surface with a kinetic energy equal to or lower than that specific value of the perpendicular kinetic energy. Consequently, for high energy values the integrated energy distribution yields one. The grey horizontal line in the bottom part of Figure 8 represents the e-folding of the proton ensemble at an integral value of $63 \%$ and is used as measure for the majority of the protons. For low power operation the majority of protons have energies below $1.4 \mathrm{eV}$ while in the high power scenario they have energies below $2.9 \mathrm{eV}$. The corresponding energy for the 1d Maxwell distribution lies - as expected - at exactly $0.8 \mathrm{eV}$.

As discussed earlier, the proton energies determined by ProtonFlow3d close to the plasma grid represent a upper limit for the energy in the experiment as Coulomb collisions and the influence of the magnetic field (but also the ambipolar force between protons and electrons) have been neglected. Thus, in the ion source prototype the energy distribution functions will be shifted even more towards lower energies. Consequently, the determined proton energies are in reasonable agreement with the $\approx 10^{4} \frac{\mathrm{m}}{\mathrm{s}}$ positive ion flow velocity (which equals a kinetic energy of $0.5 \mathrm{eV}$ for protons) determined using 
the Mach probe in a source without magnetic filter field [20].

\section{Conclusions}

A 3d Monte Carlo transport code for the transport of protons in the IPP negative ion source prototype for ITER NBI was developed in order to check whether a directed high energy positive ion beam strikes the plasma grid surface or not. This question is of high relevance since the energy of the positive ions impinging the plasma grid is directly connected to the relative relevance of the two negative hydrogen ion production channels (conversion of atoms and conversion of positive ions) as well as to the survival probability of the negative ions during their transport to the extraction apertures. The code ProtonFlow3d implements the best possible estimate, based on measurements, for the electric field of the ion source and the six most probable collision reactions for protons. The axial electric field was constructed by combining Langmuir probe measurements performed in the driver and the expansion region. As these measurements were made under different source configurations and plasma parameters the results had to be scaled in order to be implemented in the code. The calculated potential difference between driver and expansion region $(7.5 \mathrm{~V}$ and $14.7 \mathrm{~V}$ for the two investigated operation scenarios) is much smaller than the $45 \mathrm{~V}$ in [23].

As a consequence - and also due to the fact that the protons are generated with relatively low energies $\left(T\left(\mathrm{H}^{+}\right)=0.8 \mathrm{eV}\right)$ in the driver - also the mean free path for collisions (a few centimeters in the driver and up to ten centimeters in the expansion region) is much smaller than the several tens of centimeters up to meters determined in [23]. In turn, the probability for a proton to conduct a collision during the transport to the plasma grid must not be neglected: only a fraction of below one percent of the protons reaching the plasma grid are fully accelerated.

The energy distribution of the protons reaching the plasma grid are clearly different from a 1d Maxwell distribution for thermal protons with $T\left(\mathrm{H}^{+}\right)=0.8 \mathrm{eV}$. Nevertheless, they show the behavior indicative of the start of the thermalization process. If effects like Coulomb collisions, the influence of the magnetic field and ambipolar forces are considered it is expected that the proton energy distribution will be even closer to a Maxwell distribution. Altogether, the definitive statement can be drawn that no directed high energy proton beam hits the plasma grid of the IPP negative ion source prototype. Thus, the application of low temperature Maxwell distribution functions in models describing the physics of the plasma particles close to the plasma grid is reasonable.

As a consequence, it can be stated that the conversion of positive hydrogen ions to negative ions at the surface of the plasma grid is not the dominant process over the conversion of hydrogen atoms. This means that the homogeneity of the extracted negative ion beam is correlated not solely to the homogeneity of the positive hydrogen ions close to the plasma grid and the role of the hydrogen atoms must not be neglected. This finding is of particular relevance for optimizing the homogeneity of the extracted 
negative hydrogen ion beam in order to fulfill the ITER requirement.

\section{Acknowledgments}

The work was supported by a grant from Fusion for Energy (F4E-2008-GRT-007) under the responsibility of Antonio Masiello. The opinions expressed herein are those of the authors only and do not represent the Fusion for Energy's official position.

\section{References}

[1] R. Hemsworth, A. Tanga, and V. Antoni. Rev. Sci. Instrum., 79:02C109, 2008.

[2] R. Hemsworth, H. Decamps, J. Graceffa, B. Schunke, M. Tanaka, M. Dremel, A. Tanga, H.P.L. De Esch, F. Geli, J. Milnes, T. Inoue, D. Marcuzzi, P. Sonato, and P. Zaccaria. Nucl. Fusion, 49:045006, 2009.

[3] E. Speth, H. D. Falter, P. Franzen, U. Fantz, M. Bandyopadhyay, S. Christ, A. Encheva, M. Fröschle, D. Holtum, B. Heinemann, W. Kraus, A. Lorenz, Ch. Martens, P. McNeely, S. Obermayer, R. Riedl, R. Süss, R. Wilhelm A. Tanga, and D. Wünderlich. Nucl. Fusion, 46:S220, 2006.

[4] U. Fantz, P. Franzen, W. Kraus, H. D. Falter, M. Berger, S. Christ-Koch, M. Fröschle, R. Gutser, B. Heinemann, C. Martens, P. McNeely, R. Riedl, E. Speth, and D. Wünderlich. Rev. Sci. Instrum., 79:02A511, 2008.

[5] B. Heinemann, H. D. Falter, U. Fantz, P. Franzen, M. Fröschle, W. Kraus, C. Martens, R. Nocentini, R. Riedl, E. Speth, and A. Staebler. Fusion Eng. Des., 86:768, 2011.

[6] P. Franzen, U. Fantz, W. Kraus, H. Falter, B. Heinemann, R. Nocentini, and the NNBI-Team. AIP Conf. Proc., 1097:451, 2009.

[7] P. Sonato, P. Agostinetti, G. Anaclerio, V. Antoni, O. Barana, M. Bigi, M. Boldrin, M. Cavenago, S. Dal Bello, M. Dalla Palma, A. Daniele, M. DArienzo, A. De Lorenzi, A. Ferro, A. Fiorentin, E. Gaio, E. Gazza, L. Grando, F. Fantini, F. Fellin, A. Luchetta, G. Manduchi, F. Milani, D. Marcuzzi, L. Novello, R. Pasqualotto, M. Pavei, R. Pengo, S. Peruzzo, A. Pesce, N. Pilan, R. Piovan, N. Pomaro, M. Recchia, W. Rigato, A. Rizzolo, G. Serianni, M. Spolaore, P. Spolaore, S. Sandri, C. Taliercio, V. Toigo, M. Valisa, P. Veltri, P. Zaccaria, A. Zamengo, and L. Zanotto. Fusion Eng. Des., 84:269, 2009.

[8] A. Masiello, G. Agarici, T. Bonicelli, M. Simon, J. Alonso, M. Bigi, D. Boilson, G. Chitarin, C. Day, P. Franzen, S. Hanke, B. Heinemann, R. Hemsworth, A. Luchetta, D. Marcuzzi, J. Milnes, T. Minea, R. Pasqualotto, N. Pomaro, G. Serianni, W. Rigato, P. Sonato, V. Toigo, F. Villecroze, C. Waldon, and P. Zaccaria. Fusion Eng. Des., 86:860, 2011.

[9] O. Fukumasa. IEEE Trans. Plasma Sci., 28:1009, 2000.

[10] V. Dudnikov. Rev. Sci. Instrum., 73:992, 2002.

[11] B. Rasser, J. N. M. van Wunnik, and J. Los. Surf. Sci., 118:697, 1982.

[12] J. D. Isenberg, H. J. Kwon, and M. Seidl. AIP Conf. Proc., 287:38-47, 1994.

[13] R. Gutser, D. Wünderlich, U. Fantz, and the NNBI-Team. Plasma Phys. Control. Fusion, 51:045005, 2009.

[14] P. McNeely, D. Wünderlich, and the NNBI-Team. Plasma Sources Sci. Technol., 20:045005, 2011.

[15] L. Schiesko. Plasma Phys. Control. Fusion, 54:105002, 2012.

[16] U. Fantz, P. Franzen, W. Kraus, M. Berger, S. Christ-Koch, M. Fröschle, R. Gutser, B. Heinemann, Ch. Martens, P. McNeely, R. Riedl, E. Speth, and D. Wünderlich. Plasma Phys. Control. Fusion, 49:B563, 2007.

[17] D. Wünderlich, R. Gutser, and U. Fantz. Plasma Sources Sci. Technol., 18:045031, 2009.

[18] U. Fantz, P. Franzen, W. Kraus, M. Berger, S. Christ-Koch, H. Falter, M. Fröschle, R. Gutser, 
B. Heinemann, C. Martens, P. McNeely, R. Riedl, E. Speth, A. Stäbler, and D. Wünderlich. Nucl. Fusion, 49:125007, 2009.

[19] L. Schiesko, P. McNeely, U. Fantz, P. Franzen, and the NNBI-Team. Plasma Phys. Control. Fusion, 53:085029, 2011.

[20] A. Tanga, M. Bandyopadhyay, and P. McNeely. Appl. Phys. Lett., 84:182, 2004.

[21] M. Bandyopadhyay, A. Tanga, H. D. Falter, P. Franzen, B. Heinemann, D. Holtum, W. Kraus, K. Lackner, P. McNeely, R. Riedl, E. Speth, and R. Wilhelm. J. Appl. Phys., 96:4107, 2004.

[22] P. Franzen, U. Fantz, and the NNBI-Team. AIP Conf. Proc., 1390:310, 2011.

[23] M. Bacal. Rev. Sci. Instrum., 83:02B101, 2012.

[24] J. H. Whealton, E. F. Jaeger, and J. C. Whitson. Rev. Sci. Instrum., 48:829, 1977.

[25] W. S. Cooper and R. V. Pyle. National negative-ion-based neutral-beam development plan. Technical Report LBL-PUB-464, Lawrence Berkeley Lab., Berkeley, 1983.

[26] K. Makino, T. Sakurabayashi, A. Hatayama, K. Miyamoto, and M. Ogasawara. Rev. Sci. Instrum., 73:1051, 2002.

[27] P. Franzen, R. Gutser, U. Fantz, W. Kraus, H. Falter, M. Fröschle, B. Heinemann, P. McNeely, R. Nocentini, R. Riedl, A. Stäbler, and D. Wünderlich. Nucl. Fusion, 51:073035, 2011.

[28] C. K. Birdsall. IEEE Trans. on Plasma Sci., 19:65, 1991.

[29] A. E. Glassgold, P. S. Krstic, and D. R. Schultz. Astrophys. J., 621:808, 2005.

[30] T. Tabata and T. Shirai. At. Data Nucl. Data Tables, 76:1, 2000.

[31] R. K. Janev, D. Reiter, and U. Samm. Collision Proceses in Low-Temperature Hydrogen Plasmas. Technical Report JÜL-4105, Forschungszentrum Jülich, Jülich, 2003.

[32] D. Wünderlich. The CR model Yacora for molecular hydrogen, in preparation. 2012.

[33] U. Fantz, H. Falter, P. Franzen, D. Wünderlich, M. Berger, A. Lorenz, W. Kraus, P. McNeely, R. Riedl, and E. Speth. Nucl. Fusion, 46:S297, 2006.

[34] G. Herzberg. Molecular Spectra and Molecular Structure: Spectra of Diatomic Molecules. Krieger Publishing Company, Malabar, 1989.

[35] B. H. Bransden. Contemp. Phys., 31:19, 1990.

[36] T. Takizuka and H. Abe. J. Comput. Phys., 25:205, 1977.

[37] V. Vahedi and M. Surenda. Comp. Phys. Comm., 871:179, 1995.

[38] P. S. Krstic and D. R. Schultz. J. Phys. B: At. Mol. Opt. Phys., 32:2415, 1999.

[39] V. Hermann, H. Schmidt, and F. Linder. J. Phys. B: At. Mol. Phys., 11:493, 1978.

[40] D. Wünderlich. Chem. Phys., 390:75, 2011.

[41] P. McNeely, S. V. Dudin, S. Christ-Koch, U. Fantz, and the NNBI-Team. Plasma Sources Sci. Technol., 18:014011, 2009.

[42] P. Franzen, L. Schiesko, M. Fröschle, D. Wünderlich, U Fantz, and the NNBI-Team. Plasma Phys. Control. Fusion, 53:115006, 2011.

[43] S. Christ-Koch, U. Fantz, M. Berger, and the NNBI-Team. Plasma Sources Sci. Technol., 18:025003, 2009. 